\title{
Alternaria Alternata Infection in A Patient With Acute Promyelocytic Leukemia
}

\author{
Daniel Bryan Arucha, ${ }^{\mathrm{a}}$, Barry Zeluff ${ }^{\mathrm{a}}$
}

\begin{abstract}
Alternaria alternate (A. alterna) is a fungus associated with infection in immunocompromised patients. Palate and sinus infections by this organism are extremely rare but can result in significant morbidity and mortality. We present a case of a patient with acute promyelocytic leukemia with morbid nasal and palatal $A$. alternata infection.
\end{abstract}

Keywords: Alternaria; Fungal; Ascomycete; Acute promyelocytic leukemia; Opportunistic infection

\section{Introduction}

Fungal infections have become a major source of morbidity and mortality in our immunocompromised patients. As our abilities to treat and cure hematologic diseases and transplant failed organs, we continue to encounter new and rare pathology. Alternaria alternata (A. alterna) is an uncommon cause of invasive fungal infection. We report a case of nasal and sinus infection in a patient with acute promyelocytic leukemia.

\section{Case Report}

A 48 years old male presented with bruising, gingival bleed-

Manuscript accepted for publication October 21, 2011

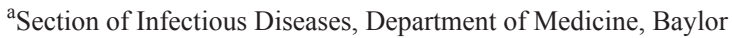
College of Medicine and St. Luke's Episcopal Hospital, Houston, Texas 77030, USA

${ }^{\mathrm{b}}$ Corresponding author: Daniel Bryan Aruch, Medicine Residency Office Baylor College of Medicine 1709 Dryden Road, Suite 5.86 Houston, TX 77030. Email: aruch@bcm.tmc.edu

doi: $10.4021 / \mathrm{jmc} 395 \mathrm{w}$ ing, and dyspnea on exertion for 2 months. He was found to have pancytopenia and transferred to our institution. He was diagnosed with acute promyelocytic leukemia (M3). He recently had noted a lesion on his right foot and his right hand, which he attributed to an unrecognized trauma along with the bruising.

His medical history included anxiety, hypertension, chronic obstructive pulmonary disease, and gastroesophageal reflux for which he took tiotropium, budesonide, formoterol, albuterol, escitalopram and omeprazole. He had a smoking history of two packs per day for thirty years. He worked on a power plant systems crew, first in a gas power plant for twenty five years and then in a coal power plant for the previous three. He denied being exposed directly to chemicals, radiation, and was not involved in digging or agricultural exposure.

Examination revealed lesions on the palate, right foot, and right index finger. After induction with idarubicin, cytarabine, and trans-retinoic acid, the lesions enlarged, corresponding with the degree of neutropenia. A computed tomogram of the sinuses, neck, and lungs demonstrated right maxillary sinus mucosal thickening and bilateral pulmonary groundglass opacities with subcentimeter indeterminate right pulmonary nodules that were not worked up further. Given his neutropenia, physical findings, and subsequent fever, he was treated with vancomycin, piperacillin/tazobactam, fluconazole, valacyclovir, and cefeprime prior to any biopsy; there was no improvement in the lesions.

Biopsy and debridement of the palate was done and showed gram-positive, filamentous bacteria in superficial layers of necrosis, which were never identified (Fig. 1A). Deeper dissection in the nasal septum and middle turbinate demonstrated necrosis and acute inflammation as well as septated hyphae, identified as A. alternata in culture (Fig. 1B). Necessary further resection resulted in the inability to swallow requiring percutaneous gastric tube placement. Biopsies of the cutaneous lesion on his index finger were obtained after treatment with amphotericin and voriconazole and demonstrated interstitial and perivascular acute inflammation with focal formation with underlying abscess and focal epidermal necrosis but no organisms were identified. He was treated 


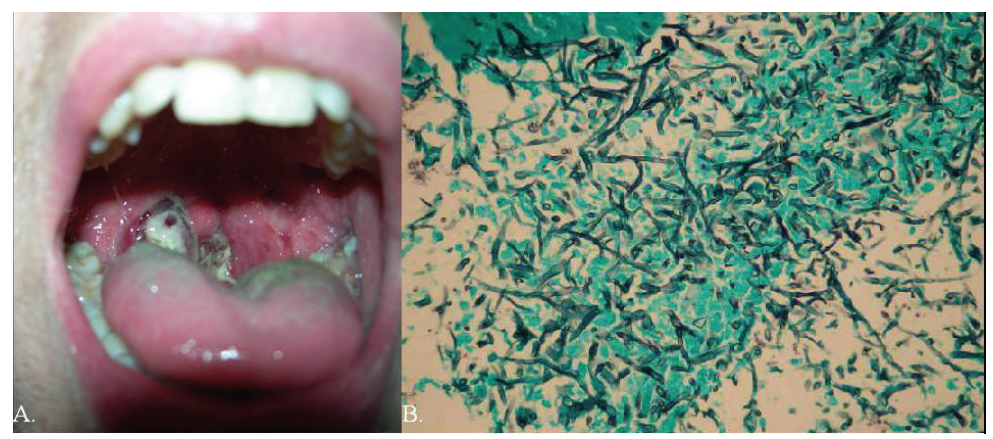

Figure 1. (A) Photograph of invasive A. alternate infection after first resection. Note hyperemic and necrotic tissue. (B) Gomori Methenamine-silver (Gms) stain showing numerous septate hyphae with $45^{\circ}$ branching.

with voriconazole and a two week course of piperacillin/ tazobactam as his neutropenia improved. Afterwards, he had clinical improvement and was discharged on indefinite voriconazole therapy.

\section{Discussion}

In the immunocompromised patient, Alternaria is recognized as a source of cutaneous, sinus, nail, palatal, and ocular infections, particularly A. alternata. Patients afflicted include those with AIDS, Cushing's disease, malignancy, and after transplant. Invasive infection requires surgical debridement as well as antifungal therapy [1-7]. Infection can result in significant morbidity, as was the case in our patient, who was no longer able to take food by mouth.

A recent review noted only 210 cases reported in the literature between 1933 and 2007, with $74 \%$ being cutaneous. Seventeen cases of invasive rhinosinusitis from $\mathrm{Al}$ ternaria were noted since 1977; only 5 had species identification, with 4 of these being A. alternata. 12/17 were immunocompromised while 10/17 had acute leukemia with neutropenia [4]. While most cases associated with hematologic malignancy occur after chemotherapy has begun, a case of cutaneous Alternaria infection at the time of acute myelogenous leukemia diagnosis has been reported $[3,7$, 8]. Our patient had evidence of palate and rhinosinusitis infection with $A$. alternata at diagnosis that worsened with initiation of chemotherapy and neutropenia. His skin biopsy did not identify any pathogens, but was obtained after beginning antifungal therapy. Therapy resulted in improvement in the cutaneous lesions, suggesting disseminated infection, although an alternative cause cannot be excluded. If Alternaria had been identified in the skin lesions or in the lesions seen on lung computed tomography, it would represent disseminated disease, which has not been described in Alternaria infection.

\section{Conflicts of Interest}

No conflicts of interest to declare.

\section{References}

1. Body BA, Sabio H, Oneson RH, Johnson CE, Kahn J, Hanna MD. Alternaria infection in a patient with acute lymphocytic leukemia. Pediatr Infect Dis J. 1987;6(4):418-420.

2. Jahagirdar BN, Morrison VA. Emerging fungal pathogens in patients with hematologic malignancies and marrow/stem-cell transplant recipients. Semin Respir Infect. 2002;17(2):113-120.

3. Neofytos D, Horn D, Anaissie E, Steinbach W, Olyaei A, Fishman J, Pfaller M, et al. Epidemiology and outcome of invasive fungal infection in adult hematopoietic stem cell transplant recipients: analysis of Multicenter Prospective Antifungal Therapy (PATH) Alliance registry. Clin Infect Dis. 2009;48(3):265-273.

4. Pastor FJ, Guarro J. Alternaria infections: laboratory diagnosis and relevant clinical features. Clin Microbiol Infect. 2008;14(8):734-746.

5. Schell WA. Unusual fungal pathogens in fungal rhinosinusitis. Otolaryngol Clin North Am. 2000;33(2):367373.

6. Segal BH, Bow EJ, Menichetti F. Fungal infections in nontransplant patients with hematologic malignancies. Infect Dis Clin North Am. 2002;16(4):935-964, vii.

7. Vieira R, Veloso J, Afonso A, Rodrigues A. Cutaneous alternariosis in a liver transplant recipient. Rev Iberoam Micol. 2006;23(2):107-109.

8. Ioannidou D, Maraki S, Kruger Krasagakis S, Stefanidou M, Krasagakis K, Alexandrakis M, Tosca A. Cutaneous alternariosis revealing acute myeloid leukaemia in an adult patient. Mycoses. 2004;47(5-6):227-230. 